\title{
ON THE SLANT HELICES ACCORDING TO BISHOP FRAME OF THE TIMELIKE CURVE IN LORENTZIAN SPACE
}

\author{
BAHADDIN BUKCU AND MURAT KEMAL KARACAN
}

Abstract. T.Ikawa obtained the following differential equation

$$
D_{T} D_{T} D_{T} T-K D_{T} T, K=\kappa^{2}-\tau^{2}
$$

for the curcular helix which corresponds the case that the curvature $\kappa$ and torsion $\tau$ of timelike curve $\alpha$ on the Lorentzian manifold $M_{1}$ are constant [5]. In this paper, we have defined a slant helix according to Bishop frame of the timelike curve. Furthermore, we have given some necessary and sufficent conditions for the slant helix and T.Ikawa's result is generalized to the case of the general slant helix.

\section{Preliminaries}

Let $M$ be an 3-dimensional smooth manifold equipped with a metric $\langle,\rangle_{L}$, where the metric $\langle,\rangle_{L}$ means a symmetric non-degenerate $(0 ; 2)$-tensor field on $M$ with constant signature. A tangent space $T_{P}(M)$ at a point $P \in M$ is furnished with the canonical inner product. If the signature of the metric $\langle,\rangle_{L}$ is $i$, then we call $M$ an indefinite-Riemannian manifold of signature $i$ and denoted by $M_{i}$. If $\langle,\rangle_{L}$ is positive definite, then $M$ is a Riemannian manifold. Especially if $i=1$, then $M$ is called a Lorentzian manifold. A tangent vector $x$ of $M_{i}$ is said to be spacelike, if $\langle x, x\rangle_{L}>0$ or $x=0$, timelike, if $\langle x, x\rangle_{L}<0$ and null or lightlike if $\langle x, x\rangle_{L}=0$ and $x \neq 0[3,4]$.

A curve in an indefinite-Riemannian manifold $M_{i}$ is a smooth mapping $\alpha: I \rightarrow M_{i}$, where $I$ is an open interval in the real line $R^{1}$. As an open submanifold of $R^{1}, I$ has a coordinate system consisting of the identity map $u$ of $I$. The velocity vector of $\alpha$ at $s \in I$

$$
\alpha^{\prime}(s)=\left.\frac{d \alpha(u)}{d u}\right|_{s} \in T_{\alpha(s)}\left(M_{i}\right) .
$$

A curve $\alpha(s)$ is said to be regular if $\alpha^{\prime}(s)$ is not equal to zero for any $s$. If $\alpha(s)$ is a spacelike or timelike curve, we can reparameterize it such that $\left\langle\alpha^{\prime}(s), \alpha^{\prime}(s)\right\rangle_{L}=1$ and $\left\langle\alpha^{\prime}(s), \alpha^{\prime}(s)\right\rangle_{L}=-1$, respectively. In this case $\alpha(s)$ is said to be unit speed or arc length parametrization $[3,4]$.

Let $R^{3}=\left\{\left(x_{1}, x_{2}, x_{3}\right): x_{1}, x_{2}, x_{3} \in R\right\}$ be a 3-dimensional vector space, and let $x=\left(x_{1}, x_{2}, x_{3}\right)$ and $y=\left(y_{1}, y_{2}, y_{3}\right)$ be two vectors in $I R^{3}$. The Lorentz scalar product of $x$ and $y$ is defined by

$$
\langle x, y\rangle_{L}=-x_{1} y_{1}+x_{2} y_{2}+x_{3} y_{3}
$$

Received April 30, 2007; revised February 18, 2008.

2000 Mathematics Subject Classification. 53A04, 53A35, 53B30.

Key words and phrases. Bishop frame, lorentzian space, parallel transport frame, slant helix, timelike curve. 
$E_{1}^{3}=\left(R^{3},\langle x, y\rangle_{L}\right)$ is called 3-dimensional Lorentzian space, Minkowski 3-Space or 3- dimensional Semi-Euclidean space.For any $x, y \in E_{1}^{3}$, Lorentzian vectoral product of $x$ and $y$ is defined by

$$
x \wedge_{L} y=\left(x_{2} y_{3}-x_{3} y_{2}, x_{1} y_{3}-x_{3} y_{1}, x_{1} y_{2}-x_{2} y_{1}\right)[2] .
$$

Let $\alpha(s)$ be a timelike curve in $M_{1}$. Denote by $\{T, N, B\}$ the moving Frenet frame along the curve $\alpha$. Then $T, N$ and $B$ are the tangent, the principal normal and binormal vectors of the curve $\alpha$ respectively. If $\alpha$ is a timelike curve, then this set of orthogonal unit vectors, known as the Frenet-Serret frame, has the following properties

$$
\begin{aligned}
\alpha^{\prime}(s) & =T \\
D_{T} T & =\kappa N \\
D_{T} N & =\kappa T+\tau B \\
D_{T} B & =-\tau N,
\end{aligned}
$$

where $D$ denotes the covariant differentiation in $M_{1}$ and $\{T, N, B\}$ are mutually orthogonal vectors satisfying the following equations

$$
\langle T, T\rangle_{L}=-1,\langle N, N\rangle_{L}=1,\langle B, B\rangle_{L}=1[8] .
$$

In a Lorentzian manifold $M_{1}$, a curve is described by the Frenet formula. For example, if all curvatures of a curve are identically zero, then the curve is a geodesic. If only the curvature $\kappa$ is a non-zero constant and the torsion $\tau$ is identically zero, then the curve is called a circle. If the curvature $\kappa$ and the torsion $\tau$ are non-zero constants, then the curve is called helix. If the curvature $\kappa$ and the torsion $\tau$ are not constant but $\frac{K}{\tau}$ is constant, then the curve is called a general helix [3, 4].

\section{Introduction}

The Bishop frame or parallel transport frame is an alternative approach to defining a moving frame that is well defined even when the timelike curve has vanishing second derivative. We can parallel transport an orthonormal frame along a timelike curve simply by parallel transporting each component of the frame. The parallel transport frame is based on the observation that, while $T(s)$ for a given curve model is unique, we may choose any convenient arbitrary basis $\left(N_{1}(s), N_{2}(s)\right)$ for the remainder of the frame, so long as it is in the normal plane perpendicular to $T(s)$ at each point. If the derivatives of $\left(N_{1}(s), N_{2}(s) t\right)$ depend only on $T(s)$ and not on each other we can make $N_{1}(s)$ and $N_{2}(s)$ vary smoothly throughout the path regardless of the curvature [1]. Therefore, we have the alternative frame equations

$$
\left[\begin{array}{l}
T^{\prime} \\
N_{1}^{\prime} \\
N_{2}^{\prime}
\end{array}\right]=\left[\begin{array}{ccc}
0 & k_{1} & k_{2} \\
k_{1} & 0 & 0 \\
k_{2} & 0 & 0
\end{array}\right]\left[\begin{array}{l}
T \\
N_{1} \\
N_{2}
\end{array}\right]
$$

where

$$
\langle T, T\rangle_{L}=-1,\left\langle N_{1}, N_{1}\right\rangle_{L}=1,\left\langle N_{2}, N_{2}\right\rangle_{L}=1 .
$$


One can show that

$$
\begin{aligned}
& \kappa(s)=\sqrt{k_{1}^{2}+k_{2}^{2}}, \\
& \theta(s)=\arctan \left(\frac{k_{2}}{k_{1}}\right), \\
& \tau(s)=\frac{d \theta(s)}{d s}
\end{aligned}
$$

[2], so that $k_{1}$ and $k_{2}$ effectively correspond to a cartesian coordinate system for the polar coordinates $\kappa, \theta$ with $\theta=\int \tau(s) d s$. The orientation of the parallel transport frame includes the arbitrary choice of integration constant $\theta_{0}$, which disappears from $\tau$ (and hence from the Frenet frame) due to the differentiation [1].

\section{The slant helices according to bishop frame of the timelike curve}

Definition 3.1. A curve $\alpha$ with $\kappa \neq 0 E^{3}$ is called a slant helix if the principal normal line of $\alpha$ make a constant angle with a fixed direction [7].

Definition 3.2. A regular timelike curve $\alpha: I \rightarrow E_{1}^{3}$ is called a slant helix provided the spacelike unit vector $N_{1}$ of the curve $\alpha$ has constant angle $\theta$ with some fixed spacelike unit vector $u$; that is, $\left\langle N_{1}(s), u\right\rangle=\cos \theta$ for all $s \in I$.

The condition is not altered by reparametrization, so without loss of generality we may assume that slant helices have unit speed. The slant helices can be identified by a simple condition on natural curvatures.

Theorem 3.1. Let $\alpha: I \rightarrow E_{1}^{3}$ be a unit speed timelike curve with non-zero natural curvatures. Then $\alpha$ is a slant helix if and only if $\frac{k_{1}}{k_{2}}$ is constant.

Proof. Let $\alpha$ is a slant helix in $E_{1}^{3}$ and $<N_{1}, u>=$ const.. Then $\alpha$ is a slant helix; from the definition we have

$$
\left\langle N_{1}, u\right\rangle=\text { const., }
$$

where the spacelike vector $u$ is a unit vector, called the axis of the slant helix. By differentiation we get

$$
\left\langle N_{1}^{\prime}, u\right\rangle=\left\langle k_{1} T, u\right\rangle=k_{1}\langle T, u\rangle=0
$$

Hence

$$
\langle T, u\rangle=0 .
$$

Again differentiating from the last equality, we can write as follows

$$
\begin{aligned}
\left\langle T^{\prime}, u\right\rangle & =\left\langle k_{1} N_{1}+k_{2} N_{2}, u\right\rangle \\
& =k_{1}\left\langle N_{1}, u\right\rangle+k_{2}\left\langle N_{2}, u\right\rangle \\
& =k_{1} \cos \theta+k_{2} \sin \theta=0 .
\end{aligned}
$$


Therefore we obtain

$$
\frac{k_{1}}{k_{2}}=-\tan \theta
$$

as desired.

Suppose that $\frac{k_{1}}{k_{2}}=-\tan \theta$. Then we can write $u \in S p\left\{N_{1}, N_{2}\right\}$, i.e.,

$$
u=N_{1} \cos \theta+N_{2} \sin \theta .
$$

Differentiating the last equality.

$$
u^{\prime}=\left(k_{1} \cos \theta+k_{2} \sin \theta\right) T=0 .
$$

So the spacelike vector $u$ is a constant vector. Thus, the proof is done.

Theorem 3.2. Let $\alpha: I \rightarrow E_{1}^{3}$ be a unit speed timelike curve.Then $\alpha$ is a slant helix iff

$$
\operatorname{det}\left(N_{1}^{\prime}, N_{1}^{\prime \prime}, N_{1}^{\prime \prime \prime}\right)=0
$$

Proof. ( $\Rightarrow$ :) Suppose that $\frac{k_{1}}{k_{2}}$ be constant. We have equalities as

$$
\begin{aligned}
& N_{1}^{\prime}=k_{1} T \\
& N_{1}^{\prime \prime}=k_{1}^{\prime} T+k_{1}\left(k_{1} N_{1}+k_{2} N_{2}\right)=k_{1}^{\prime} T+k_{1}^{2} N_{1}+k_{1} k_{2} N_{2} \\
& N_{1}^{\prime \prime \prime}=k_{1}^{\prime \prime} T+k_{1}^{\prime}\left(k_{1} N_{1}+k_{2} N_{2}\right)+2 k_{1} k_{1}^{\prime} N_{1}+k_{1}^{2}\left(k_{1} T\right)+k_{1}^{\prime} k_{2} N_{2}+k_{1} k_{2}^{\prime} N_{2}+k_{1} k_{2}\left(k_{2} T\right) \\
& N_{1}^{\prime \prime \prime}=\left(k_{1}^{\prime \prime}+k_{1}^{3}+k_{1} k_{2}^{2}\right) T+\left(3 k_{1} k_{1}^{\prime}\right) N_{1}+\left(2 k_{1}^{\prime} k_{2}+k_{1} k_{2}^{\prime}\right) N_{2}
\end{aligned}
$$

So we get

$$
\begin{aligned}
\operatorname{det}\left(N_{1}^{\prime}, N_{1}^{\prime \prime}, N_{1}^{\prime \prime \prime}\right) & =k_{1}^{2}\left[\begin{array}{ccc}
1 & 0 & 0 \\
* & k_{1} & k_{2} \\
\star & 3 k_{1} k_{1}^{\prime} & 2 k_{1}^{\prime} k_{2}+k_{1} k_{2}^{\prime}
\end{array}\right] \\
\operatorname{det}\left(N_{1}^{\prime}, N_{1}^{\prime \prime}, N_{1}^{\prime \prime \prime}\right) & =k_{1}^{2}\left(2 k_{1}^{\prime} k_{1} k_{2}+k_{1}^{2} k_{2}^{\prime}-3 k_{1} k_{1}^{\prime} k_{2}\right) \\
& =k_{1}^{3}\left(k_{2}^{\prime} k_{1}-k_{2} k_{1}^{\prime}\right) \\
& =k_{1}^{3}\left(k_{1}^{\prime} k_{2}-k_{1} k_{2}^{\prime}\right) \\
& =k_{1}^{3}\left[\frac{k_{1}^{\prime} k_{2}-k_{1} k_{2}^{\prime}}{k_{2}^{2}}\right] k_{2}^{2} \\
& =k_{1}^{3} k_{2}^{2}\left(\frac{k_{1}}{k_{2}}\right)^{\prime}
\end{aligned}
$$

Since $\alpha$ is a slant helix, $\frac{k_{1}}{k_{2}}$ is constant. Hence, we have

$$
\operatorname{det}\left(N_{1}^{\prime}, N_{1}^{\prime \prime} N_{1}^{\prime \prime \prime}\right)=0, k_{2} \neq 0 .
$$


$(\Leftarrow:)$ Suppose that $\operatorname{det}\left(N_{1}^{\prime}, N_{1}^{\prime \prime} N_{1}^{\prime \prime \prime}\right)=0$. Then it is clear that the $\frac{k_{1}}{k_{2}}=$ const. since $\left(\frac{k_{1}}{k_{2}}\right)^{\prime}$ is zero.

Theorem 3.3 Let $\alpha: I \rightarrow E_{1}^{3}$ be a unit speed timelike curve. Then $\alpha$ is a slant helix iff

$$
\operatorname{det}\left(N_{2}^{\prime}, N_{2}^{\prime \prime}, N_{2}^{\prime \prime \prime}\right)=0
$$

Proof. ( $\Rightarrow$ :) Suppose that $\frac{k_{1}}{k_{2}}$ be constant. From eq. (2.1) one can find

$$
N_{2}^{\prime}=k_{2} T
$$

and

$$
\begin{aligned}
N_{2}^{\prime \prime} & =k_{2}^{\prime} T+k_{2} T^{\prime}=k_{2}^{\prime} T+k_{2}\left(k_{1} N_{1}+k_{2} N_{2}\right)=\left(k_{2}^{\prime}\right) T+\left(k_{1} k_{2}\right) N_{1}+\left(k_{2}^{2}\right) N_{2} \\
N_{2}^{\prime \prime \prime} & =\left(k_{2}^{\prime \prime} T+k_{2}^{\prime} T^{\prime}\right)+\left(k_{1}^{\prime} k_{2} N_{1}+k_{1} k_{2}^{\prime} N_{1}+k_{1} k_{2} N_{1}^{\prime}\right)+\left(2 k_{2} k_{2}^{\prime} N_{2}+k_{2}^{2} N_{2}^{\prime}\right) \\
& =k_{2}^{\prime \prime} T+k_{2}^{\prime}\left(k_{1} N_{1}+k_{2} N_{2}\right)+k_{1}^{\prime} k_{2} N_{1}+k_{1} k_{2}^{\prime} N_{1}+k_{1} k_{2}\left(k_{1} T\right)+2 k_{2} k_{2}^{\prime} N_{2}+k_{2}^{2}\left(k_{2} T\right) \\
& =k_{2}^{\prime \prime} T+k_{1} k_{2}^{\prime} N_{1}+k_{2} k_{2}^{\prime} N_{2}+k_{1}^{\prime} k_{2} N_{1}+k_{1} k_{2}^{\prime} N_{1}+k_{1}^{2} k_{2} T+2 k_{2} k_{2}^{\prime} N_{2}+k_{2}^{3} T \\
& =\left(k_{2}^{\prime \prime}+k_{1}^{2} k_{2}+k_{2}^{3}\right) T+\left(2 k_{1} k_{2}^{\prime}+k_{1}^{\prime} k_{2}\right) N_{1}+\left(3 k_{2} k_{2}^{\prime}\right) N_{2} .
\end{aligned}
$$

Moreover, we have

$$
\begin{aligned}
\operatorname{det}\left(N_{2}^{\prime}, N_{2}^{\prime \prime} N_{2}^{\prime \prime \prime}\right) & =k_{2}^{2}\left[\begin{array}{ccc}
1 & 0 & 0 \\
\Delta & k_{1} & k_{2} \\
4 & 2 k_{1} k_{2}^{\prime}+k_{1}^{\prime} k_{2} & 3 k_{2} k_{2}^{\prime}
\end{array}\right] \\
& =k_{2}^{2}\left(3 k_{1} k_{2} k_{2}^{\prime}-2 k_{1} k_{2}^{\prime} k_{2}-k_{1}^{\prime} k_{2} k_{2}\right) \\
& =-k_{2}^{3}\left(k_{1}^{\prime} k_{2}-k_{1} k_{2}^{\prime}\right) \\
& =-k_{2}^{3}\left[\frac{k_{1}^{\prime} k_{2}-k_{1} k_{2}^{\prime}}{k_{2}^{2}}\right] k_{2}^{2} \\
& =-k_{2}^{5} \operatorname{Big}\left(\frac{k_{1}}{k_{2}}\right)^{\prime} .
\end{aligned}
$$

Since $\alpha$ is a slant helix curve $\frac{k_{1}}{k_{2}}$ is constant. Hence, we have

$$
\operatorname{det}\left(N_{2}^{\prime}, N_{2}^{\prime \prime} N_{2}^{\prime \prime \prime}\right)=0, k_{2} \neq 0
$$

$\left(\Leftarrow\right.$ :) Suppose that $\operatorname{det}\left(N_{2}^{\prime}, N_{2}^{\prime \prime} N_{2}^{\prime \prime \prime}\right)=0$. Then it is clear that the $\frac{k_{1}}{k_{2}}=$ const. $\operatorname{since}\left(\frac{k_{1}}{k_{2}}\right)^{\prime}$ is zero.

Next we consider general slant helices in the Lorentzian manifold $M_{1}$. Then we have equalities

$$
\left\{\begin{array}{c}
\alpha^{\prime}(s)=T \\
D_{T} T=k_{1} N_{1}+k_{2} N_{2} \\
D_{T} N_{1}=k_{1} T \\
D_{T} N_{2}=k_{2} T
\end{array}\right.
$$


for any $s \in I$, where $N_{1}$ and $N_{2}$ are vector fields and $k_{1}$ and $k_{2}$ are functions of parameter $s$.

Theorem 3.4. Let $\alpha: I \rightarrow E_{1}^{3}$ be a unit speed timelike curve on $M_{1}$ is a general slant helix iff

$$
D_{T}\left(D_{T} D_{T} N_{1}\right)=A D_{T} N_{1}+3 k_{1}^{\prime} D_{T} T .
$$

where

$$
A=\kappa^{2}+\frac{k_{1}^{\prime \prime}}{k_{1}}, \quad k_{1}^{2}+k_{2}^{2}=\kappa^{2}
$$

Proof. Suppose that $\alpha$ is a general slant helix. Then, from (3.1), we have

$$
\begin{aligned}
D_{T}\left(D_{T} N_{1}\right) & =D_{T}\left(k_{1} T\right)=k_{1}^{\prime} T+k_{1} D_{T} T \\
& =k_{1}^{\prime} T+k_{1}\left(k_{1} N_{1}+k_{2} N_{2}\right) \\
D_{T}\left(D_{T} N_{1}\right) & =k_{1}^{\prime} T+k_{1}^{2} N_{1}+k_{1} k_{2} N_{2}
\end{aligned}
$$

and

$$
\begin{gathered}
D_{T}\left(D_{T} D_{T} N_{1}\right)=k_{1}^{\prime \prime} T+k_{1}^{\prime} D_{T} T+2 k_{1} k_{1}^{\prime} N_{1}+k_{1}^{2} D_{T} N_{1}+k_{1}^{\prime} k_{2} N_{2}+k_{1} k_{2}^{\prime} N_{2}+k_{1} k_{2}\left(k_{2} T\right) \\
D_{T}\left(D_{T} D_{T} N_{1}\right)=k_{1}^{\prime \prime} T+k_{1}^{\prime} D_{T} T+2 k_{1} k_{1}^{\prime} N_{1}+k_{1}^{2} D_{T} N_{1}+k_{1}^{\prime} k_{2} N_{2}+k_{1} k_{2}^{\prime} N_{2}+k_{1} k_{2}^{2} T \\
D_{T}\left(D_{T} D_{T} N_{1}\right)=\left(k_{1}^{\prime \prime}+k_{1} k_{2}^{2}\right) T+k_{1}^{2} D_{T} N_{1}+2 k_{1} k_{1}^{\prime} N_{1}+\left(k_{1}^{\prime} k_{2}+k_{1} k_{2}^{\prime}\right) N_{2}+k_{1}^{\prime} D_{T} T
\end{gathered}
$$

Now, since $\alpha$ is a general slant helix, we have

$$
\frac{k_{1}}{k_{2}}=\text { constant }
$$

and this upon the derivation gives rise to

$$
k_{1}^{\prime} k_{2}=k_{1} k_{2}^{\prime} .
$$

If we substitute the values

$$
T=\frac{1}{k_{1}} D_{T} N_{1}
$$

and

$$
\left(k_{1} k_{2}\right)^{\prime}=2 k_{1}^{\prime} k_{2} \text {, }
$$

in (3.4) we obtain

$$
\begin{aligned}
D_{T}\left(D_{T} D_{T} N_{1}\right) & =\left(k_{1}^{\prime \prime}+k_{1} k_{2}^{2}\right)\left(\frac{1}{k_{1}} D_{T} N_{1}\right)+k_{1}^{2} D_{T} N_{1}+\left(2 k_{1} k_{1}^{\prime} N_{1}+2 k_{1}^{\prime} k_{2} N_{2}\right)+k_{1}^{\prime} D_{T} T \\
& =\left(\frac{k_{1}^{\prime \prime}}{k_{1}}+k_{2}^{2}\right) D_{T} N_{1}+k_{1}^{2} D_{T} N_{1}+\left(2 k_{1} k_{1}^{\prime} N_{1}+2 k_{1}^{\prime} k_{2} N_{2}\right)+k_{1}^{\prime} D_{T} T
\end{aligned}
$$




$$
\begin{aligned}
= & \left(\frac{k_{1}^{\prime \prime}}{k_{1}}+\kappa^{2}\right) D_{T} N_{1}+2 k_{1}^{\prime}\left(k_{1} N_{1}+k_{2} N_{2}\right)+k_{1}^{\prime} D_{T} T \\
= & \left(\frac{k_{1}^{\prime \prime}}{k_{1}}+\kappa^{2}\right) D_{T} N_{1}+2 k_{1}^{\prime} D_{T} T+k_{1}^{\prime} D_{T} T \\
D_{T}\left(D_{T} D_{T} N_{1}\right)= & \left(\frac{k_{1}^{\prime \prime}}{k_{1}}+\kappa^{2}\right) D_{T} N_{1}+3 k_{1}^{\prime} D_{T} T . \\
& D_{T}\left(D_{T} D_{T} N_{1}\right)-\left(\kappa^{2}+\frac{k_{1}^{\prime \prime}}{k_{1}}\right) D_{T} N_{1}=3 k_{1}^{\prime} D_{T} T .
\end{aligned}
$$

So we get as desired.

Conversely let us assume that (3.1) holds. We show that the timelike curve $\alpha$ is a general slant helix. Differentiating covariantly (3.5) we obtain

$$
\begin{aligned}
& D_{T} T=D_{T}\left(\frac{1}{k_{1}} D_{T} N_{1}\right) \\
& D_{T} T=-\frac{k_{1}^{\prime}}{k_{1}^{2}} D_{T} N_{1}+\frac{1}{k_{1}} D_{T} D_{T} N_{1}
\end{aligned}
$$

and so,

$$
D_{T} D_{T} T=\left(-\frac{k_{1}^{\prime}}{k_{1}^{2}}\right)^{\prime} D_{T} N_{1}-\frac{k_{1}^{\prime}}{k_{1}^{2}} D_{T} D_{T} N_{1}-\frac{k_{1}^{\prime}}{k_{1}^{2}} D_{T} D_{T} N_{1}+\frac{1}{k_{1}} D_{T} D_{T} D_{T} N_{1}
$$

If we use (3.1) in (3.6), we get

$$
\begin{gathered}
D_{T} D_{T} T=\left(-\frac{k_{1}^{\prime}}{k_{1}^{2}}\right)^{\prime} D_{T} N_{1}-\frac{2 k_{1}^{\prime}}{k_{1}^{2}} D_{T} D_{T} N_{1}+\frac{1}{k_{1}} A D_{T} N_{1}+\frac{3 k_{1}^{\prime}}{k_{1}} D_{T} T \\
=\left[\left(-\frac{k_{1}^{\prime}}{k_{1}^{2}}\right)^{\prime}+\frac{A}{k_{1}}\right] D_{T} N_{1}-\frac{2 k_{1}^{\prime}}{k_{1}^{2}} D_{T} D_{T} N_{1}+\frac{3 k_{1}^{\prime}}{k_{1}} D_{T} T \\
D_{T} D_{T} T=\left[\left(-\frac{k_{1}^{\prime}}{k_{1}^{2}}\right)^{\prime}+\frac{A}{k_{1}}\right] D_{T} N_{1}-\frac{2 k_{1}^{\prime}}{k_{1}^{2}}\left(k_{1}^{\prime} T+k_{1}^{2} N_{1}+k_{1} k_{2} N_{2}\right)+\left(\frac{3 k_{1}^{\prime}}{k_{1}} k_{1} N_{1}+\frac{3 k_{1}^{\prime}}{k_{1}} k_{2} N_{2}\right) \\
=\left[\left(-\frac{k_{1}^{\prime}}{k_{1}^{2}}\right)^{\prime}+\frac{A}{k_{1}}\right] D_{T} N_{1}-2\left(\frac{k_{1}^{\prime}}{k_{1}}\right)^{2} T-2 k_{1}^{\prime} N_{1}-\frac{2 k_{1}^{\prime} k_{2}}{k_{1}} N_{2}+3 k_{1}^{\prime} N_{1}+\frac{3 k_{1}^{\prime} k_{2}}{k_{1}} N_{2} .
\end{gathered}
$$

Substituting (3.3) and (3.4) in this last equality we have

$$
D_{T} D_{T} T=\left[\left(-\frac{k_{1}^{\prime}}{k_{1}^{2}}\right)^{\prime}+\frac{A}{k_{1}}\right] D_{T} N_{1}-2\left(\frac{k_{1}^{\prime}}{k_{1}}\right)^{2} T+k_{1}^{\prime} N_{1}+\frac{k_{1}^{\prime} k_{2}}{k_{1}} N_{2} .
$$

On the other hand we can write $D_{T}\left(D_{T} T\right)$ as follows

$$
D_{T}\left(D_{T} T\right)=k_{1} D_{T} N_{1}+k_{2}^{2} T+k_{1}^{\prime} N_{1}+k_{2}^{\prime} N_{2}
$$


From comparision the (3.7) and (3.8) we obtain the equalities below

$$
\frac{k_{1}^{\prime} k_{2}}{k_{1}}=k_{2}^{\prime}
$$

and so

$$
\frac{k_{1}^{\prime}}{k_{2}}=\frac{k_{2}^{\prime}}{k_{2}}
$$

Integrating (3.9), we get

$$
\frac{k_{1}}{k_{2}}=\text { const }
$$

Thus $\alpha$ is a general slant helix. Hence, the proof is done.

\section{References}

[1] L.R. Bishop, There is more than one way to frame a curve, Amer. Math. Monthly 82(1975), 246-251.

[2] B. Bukcu and M. K. Karacan, Bishop Frame of The Timelike Curve in Minkowski 3-Space, Süleyman Demirel University,Faculty of Science and Art, Journal of Science, (Accepted for Publication) 2008.

[3] N. Ekmekci and H. H. Hacisalihoglu, On helices of a lorentzian manifold, Commun. Fak. Sci. Univ. Ankara, Series A1 45(1996), 4-5-50.

[4] K. Ilarslan, Characterizations of spacelike general helices in Lorentzian manifold, Kragujevac J. 25(2003), 209-218.

[5] T. Ikawa, On Curves and Submanifolds in an Indefinite-Riemannian Manifold, Tsukuba J. Math. 9(1985), 353-371.

[6] J. Oprea, Differential Geometry and Its Applications, Prentice Hall, N.J. 07458, 1997.

[7] L. Kula and Y. Yayli, On slant helix and its spherical indicatrix, Applied Mathematics and Computation 169(2005), 600-607.

[8] M. Petrovic-Torgasev and E. Sucurovic, Some characterizations of the lorentzian spherical timelike and null, Matematiqki Vesnik, 53(2001), 21-27.

Gazi Osman Pasa University, Faculty of Sciences and Arts, Department of Mathematics,Tokat-TURKEY. E-mail: bbukcu@yahoo.com, bbukcu@yahoo.com

Usak University, Faculty of Sciences and Arts, Department of Mathematics, 1 Eylul Campus, 64200, UsakTURKEY.

E-mail: murat.karacan@usak.edu.tr 